\title{
COMPARATIVE STUDY OF DIFFERENT KALMAN FILTER IMPLEMENTATIONS IN POWER SYSTEM STABILITY
}

\author{
${ }^{1}$ Goh, H.H., ${ }^{1}$ C.W. Tai, ${ }^{1}$ Q.S. Chua, ${ }^{1}$ S.W. Lee, \\ ${ }^{1}$ B.C. Kok, ${ }^{2}$ K.C. Goh and ${ }^{3}$ K.T.K. Teo \\ ${ }^{1}$ Departmentof Electrical Power Engineering, Faculty of Electrical and Electronic Engineering, \\ ${ }^{2}$ Departmentof Construction Management, Faculty of Technology Management and Business, \\ Universiti Tun Hussein Onn Malaysia, 86400 Parit Raja, BatuPahat, Johor, Malaysia \\ ${ }^{3}$ Modelling, Simulation and Computing Laboratory, Level 3, Block C, \\ School of Engineering and Information Technology, Jalan UMS, \\ Universiti Malaysia Sabah, 88400 Kota Kinabalu, Sabah, Malaysia
}

Received 2014-04-08; Revised 2014-04-12; Accepted 2014-05-29

\begin{abstract}
Voltage stability and voltage collapse issues have in recent years begun to constitute an unpleasant warning to the operational security of power systems. Many techniques have been investigated in order to predict the point of voltage collapse. However, there are still several restrictions due to the insufficiency of current system state information. Accompanied by the commencement of the Phasor Measurement Units (PMUs) evolving technology, it donates a solution to enhance the existing power system state estimation. In consequence, the significances to develop preferable methods that would provide a preliminary warning before the voltage collapse had grabbed the attention. This study covers the forming of real-time system monitoring methods that able to provide a timely warning in the power system. The algorithms used to estimate the points of collapse are according to the theory that voltage instability is approximately linked to the maximum load ability of a transmission network. As a result, the critical operating conditions (peak of maximum deliverable power) come when the system Thevenin impedance is equal to the load impedance. This study focuses specifically on research about the motivation and the application of different Kalman filter implementations such as Discrete Kalman Filter (DKF), Extended Kalman Filter (EKF) and Unscented Kalman Filter (UKF) are used to track the Thevenin parameters. Therefore, the implications of this research paper are to determine the robustness and reliability of the proposed tracking methods. As compared to previous studies, the tracking process is just mainly focused on DKF method only, while the novelty throughout this study is to compare the performances and efficiencies of different Kalman filters in determining the maximum load ability on the 2 different types of test systems. Accompanying, the parameters are utilized in real-time voltage instability estimator to discover the current system's condition. In this study, the effectiveness of the proposed algorithms is assessed under a large number of random operating conditions on the Malaysia's power system $132 \mathrm{kV}, 2$-bus and 10-bus systems. Eventually, the results are differentiated by using the early-warning index of voltage collapse. All through the test cases, EKF method shows the best ability to track the Thevenin parameters as compared to DKF and UKF. Last but not least, the earlywarning index acted as a pioneer implication in estimating the maximum load power ability of the power system right before load shedding methods are being executed.
\end{abstract}

Keywords: Discrete Kalman Filter (DKF), Extended Kalman Filter (EKF), Point of Collapse, Real Time Monitoring System, Thevenin Parameters, Unscented Kalman Filter (UKF), Voltage Collapse, Voltage Instability

Corresponding Author: Goh, H.H., Department of Electrical Power Engineering, Faculty of Electrical and Electronic Engineering, Universiti Tun Hussein Onn Malaysia, 86400 Parit Raja, BatuPahat, Johor, Malaysia 


\section{INTRODUCTION}

Voltage stability has been an anxious curiosity of industry and research sectors widely around the globe. As the effect of the load growth without a relative increase of transmission capacity has contributed many power systems operating closer to the limit in conjunction with the network expansion is restricted due to many reasons such as limited expension and high investment expenses. From this ground, a continuous real-time system monitoring based on the voltage instability predictor is required to calculate the length of the peak maximum deliverable power.

Phasor Measurement Units (PMUs) technology is able to produce a shot of the current system state by measuring such parameters as positive sequence voltage and current magnitude and angle and frequency. The Malaysia power system is equipped with Phasor Measurement Units (PMUs). The implementation of the PMU technology in real power systems is in progress currently. In accordance with the announcement on the increment in electricity tariff from (TNB, 2011), the continually increasing in load demand and fuel cost leads to an urgent need to improve operational efficiency's utilization of transmission and distribution assets and reduction of losses.

This research study was analysing data collected from PMUs system and different Kalman methods for voltage stability analysis are implemented. Voltage collapse such as fault occurrence or tripping on the bus will be created by referring to the simulation data from the Real-Time Digital Simulator (RTDS). The PMUs simulated data is held in the form of electric potential and current phasors which refer to its magnitude and angle. For the time being, Tenaga Nasional Berhad Research (TNB-R) is using the PMUs technology that's able to measure 50 samples per second. The experimental performance of simulation was conducted at TNB-R with the $132 \mathrm{kV}$ load area under investigation. The limitation of this project is due to the communication channels at some TNB substations of interest are not fully configured, thus the measurements based on real data streams are still impossible at the second. Therefore, the results shown are based on input from simulations.

In this study, three different types of Kalman filter implementations such as DKF, EKF and UKF are being taken to track the Thevenin equivalent by using the voltage and current provided from PMUs. Then, the Thevenin equivalent is used to calculate the early warning index to monitor the condition of the power system.
Furthermore, the Thevenin equivalent is also applied to compute the maximum power transfer to determine a voltage stability index. The Voltage Stability Margin (VSM) can be expressed in terms of active power, reactive power and apparent power (Chen et al., 2012). With the VSM index achieved, the decision making can be done easily either provide information to the operator for further control actions.

The rest of this study is organized as follows. A background study on the overview of voltage stability assessment, optimal PMUs placement and significance and preceding works on DKF, EKF and UKF will be discussed in section 2. Distinct Kalman filters accomplishments will be talked about in section 3. Results and discussion will be comprised in section 4. Finally, this study is concluded together with the future work in section 5 .

\section{BACKGROUND OF STUDY}

\subsection{Voltage Stability Assessment}

Voltage stability is the ability to maintain the voltage at an acceptable level under normal conditions. Voltage stability is closely related to the notion of maximum load ability of a transmission network. Merited by voltage stability characteristics (10 sec to a few minute's ranges of time periods) (Kundur et al., 2004), voltage stability of a stressed power system should be supervised in real-time to authorize suitable control measures effort in a timely manner. Apart from that, voltage instability, perhaps leads to voltage collapse in the system or subsystem. Voltage stability is continuous issues despite various researchers were conducted a couple of decades ago.

Various events commenced by voltage instability and induced to voltage collapse were reported in many regions of the world (Lee and Lee, 1991). One of the local issues experienced in Malaysia was the major blackout event in 13 January 2005 and ultimately enforced the local authority to shed some of the load to balance the demands (Vaiman et al., 2012).

Voltage instability commencement is caused by the electrical distance between generations and loads and depends on the topology of the network. Besides, power system affected by disturbances can enter a state of voltage instability after a progressive and uncontrollable drop in voltage (Chakrabarti and Halder, 2006).

The predictions of voltage stability and proximity to voltage collapse have been advised to take steps before corrective actions such as the load shedding measures. A number of formerly works have been established to predict the voltage stability and the proximity to voltage collapse. The prediction initiated with conventional 
approaches, for example, PV and QV curves and sensitivity based indices (Kundur, 1994). Following by continuation methods with the special advantage of being able to converge even near the nose points of the QV curves, where other load flow programs fail to converge (Ajjarapu and Meliopoulos, 2008; Balasubramanian and Singh, 2011). These methods provide accurate and complete results, but they are inhibited because of consuming longer computing time due to the repetitive power flow calculations.

\subsection{Optimal PMUs Placement and Significance}

The optimum PMUs placement methods with applications to state estimation accuracy have been hashed out in (Zhu et al., 2009). The authors have considered the geographic location of the PMUs within the power system naturally affects the value added by PMUs to one specific application in power grid control and operation. The analysis indicates that there are considerable gains to be made in estimator accuracy if the PMUs are placed in accordance with the method when compared to the current placement strategy. It could be observed when the PMUs monitored buses reach a certain number in the entire network; the further introduction of phasor measurements will not be as effective in the estimation accuracy improvement.

The estimator determines the best approximation of the current power system states, normally including the voltage phasors, transformer taps positions and circuit breaker status, moved over the stream of telemetry that has been gathered from the system's sensors, current network model and information from other data sources. In the modern control centres, estimates are the major input for many grid supervision applications. Thus, state estimator is commonly referred as the "boarding ticket" to many other power system monitoring and control applications (Zhu et al., 2009). The illustration of the role of state estimation in power system control and operation is shown in Fig. 1.

\subsection{Preceding Works on DKF, EKF and UKF}

Several works were purported to identify the voltage instability in a specified load bus for a power system (Vu et al., 2001; Al-Ammari, 2002; Min et al., 2009). They are based on the DKF algorithm and the maximum power transfer principle. The proposed technique is used to determine Thevenin equivalent of a bus for different loading conditions, either when the total system load's changes at the same rate (long-term voltage stability problem) or when a load on a certain bus change. The proposed algorithm employs the real measurements at the bus to calculate the load impedance. These measurements are the load voltage and current. Thus, it can be implemented on-line on the control centres to investigate the voltage stability. DKF algorithm is being used to implement to identify the critical point beyond which voltage instability may occur. The results obtained in this study show that DKF algorithm is an effective algorithm for measuring Thevenin impedance for short-term and long-term voltage stability studies (Al-Ammari, 2002).

The State-of-Charge Estimation of the Lithium-Ion Battery Using an Adaptive Extended Kalman Filter (EKF). EKF algorithm is able to obtain a better convergent and robust result that can greatly improve the dependence of the traditional filter algorithm on the battery model. The typical characteristics of the lithiumion battery are analyzed by experiment. An improved Thevenin battery model is achieved and model parameters are identified by using the EKF algorithm. There is a comparison between the EKF algorithm and the adaptive EKF and yet the results for adaptive EKF, which present that the estimation algorithm has higher accuracy compared with the EKF algorithm. Ordinarily, the EKF algorithm possesses certain stability; even so, it easily causes divergence due to the error existing in the model and the noise statistics, particularly the noise statistics evaluated by the trial-and-error method (He et al., 2011).

An investigation based on online state estimation of the synchronous generator by using the Unscented Kalman Filter was introduced (Ghahremani and Kamwa, 2011). The authors presented an online state estimator in the model based category, according to above classification, uses the UKF algorithm to generate the estimated states from the available signals obtained from PMUs, which is assumed to be installed in the substation of a power plant.

As a conclusion from the previous studies, the UKF addressed the approximation issues of the EKF. The state distribution is again represented by a Gaussian Random Variable (GRV), but is specified using a minimal set of carefully chosen sample points. These points completely capture the true mean and covariance of the GRV. The UKF consistently achieves a safer grade of better accuracy than the EKF at a comparable level of complexity. In EKF, the state distribution is propagated analytically through the first-order linearization of the nonlinear system due to which; the posterior mean and covariance could be corrupted. The UKF which is a derivative-free alternative to EKF can surmount this trouble by using a deterministic sampling approach. The state distribution is represented using a minimal set of carefully chosen sample points, called sigma points. 


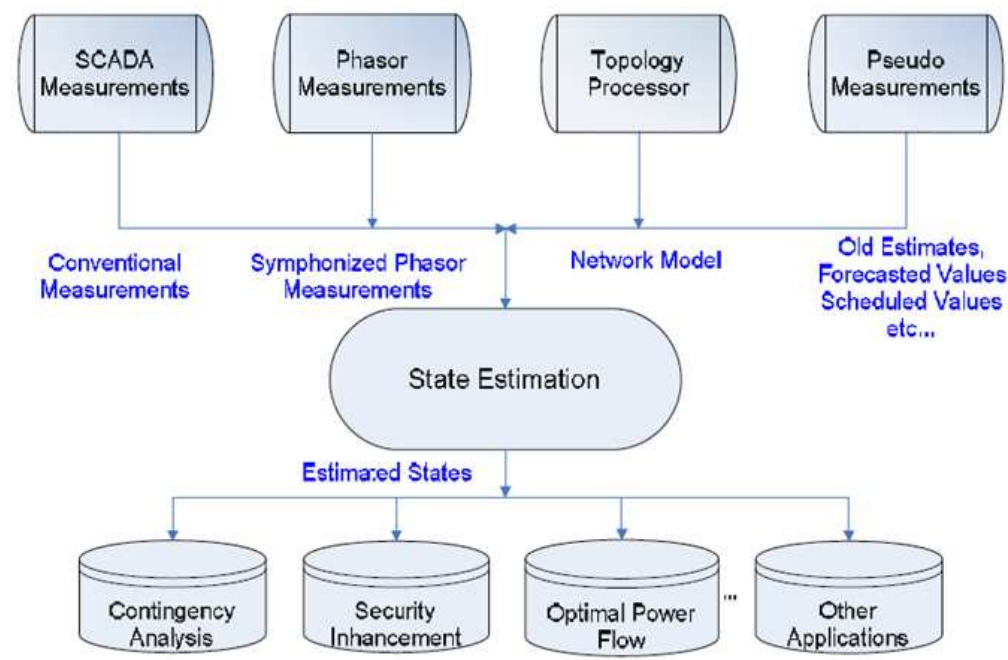

Fig. 1. Contribution of state estimation in power system control and operation (Wu and Giri, 2006)

Same as in the EKF, UKF consists of the same two steps which are the model forecast and data assimilation, except they are now preceded by another step for the selection of sigma points.

\section{DISTINCT KALMAN FILTERS ACCOMPLISHMENTS}

\subsection{Tracking Thevenin Equivalent}

Numbers of techniques have been aimed to track the Thevenin equivalent. This step is requisite for acquiring two indicators which will be discussed in the following sections.

Based on Fig. 2, bus $\mathrm{Z}$ has a load demand on the right side. Besides, the Thevenin equivalent of the system (the rest of the power system) is connected to the left side of the bus $\mathrm{Z}$ as shown in Fig. 2.

The voltage equation at bus $\mathrm{Z}$ during time $\mathrm{t}$ taken from the measurement in Fig. 2 can be expressed as:

$$
\mathrm{Eth}=\mathrm{V}+(\mathrm{Zth} * \mathrm{I})
$$

Partition Equation 1 into two equations of real and imaginary parts, then indirectly notice that at least two sets of voltage and current information about the bus $\mathrm{Z}$ at time $\mathrm{t}$ are required to solve the equation. By using above formulations, Equation 1 can be altered to:

$$
\left[\begin{array}{cccc}
1 & 0 & -\mathrm{Ir} & \mathrm{Ii} \\
0 & 1 & -\mathrm{Ii} & -\mathrm{Ir}
\end{array}\right] *\left[\begin{array}{l}
\mathrm{E} \text { thr } \\
\text { E thi } \\
\mathrm{R} \text { th } \\
\mathrm{X} \text { th }
\end{array}\right]=\left[\begin{array}{c}
\mathrm{Vr} \\
\mathrm{Vi}
\end{array}\right] \equiv(\mathrm{H} * \hat{\mathrm{X}})+\mathrm{v}=\hat{\mathrm{Z}}
$$

Where:

$\mathrm{E}_{\text {thr }}=$ The real part of the Thevenin equivalent voltage

$\mathrm{E}_{\mathrm{thi}} \quad=$ The imaginary of the Thevenin equivalent voltage

$\mathrm{R}_{\mathrm{th}} \quad=$ The resistive part of Thevenin equivalent impedance

$\mathrm{X}_{\mathrm{th}} \quad=$ The reactive part of Thevenin equivalent impedance

$\mathrm{V}_{\mathrm{r}}$ and $\mathrm{V}_{\mathrm{i}}=$ The real and imaginary parts of load voltage $I_{r}$ ans $I_{i} \quad=$ The real and imaginary parts of load current

In this study, two measurements which are load Voltage (V) and load current (I) taken at time tare obtained from the Real-Time Digital Simulator (RTDS). The unknown are $\mathrm{R}_{\mathrm{th}}, \mathrm{X}_{\mathrm{th}}, \mathrm{E}_{\mathrm{thr}}, \mathrm{E}_{\mathrm{thi}}$. In Equation 2, there are two equations and four unknowns, so clearly measurements taken at two or more different times are required to solve for unknowns. In a real environment, measurements are not precise and the Thevenin parameters drift due to the system's changing conditions. To suppress oscillations, a larger data window needs to be used. The estimation, therefore, attempts to minimize the error in a least-square sense.

\subsection{Proposed Kalman Filter Implementations}

\subsubsection{Discrete Kalman Filter (DKF)}

The DKF estimates a process by using a sort of feedback control. The filter estimates the process state at some time and then obtains feedback in the form of noisy measurements. As such, the equations for the DKF contain two groups, which are the time update equations and the measurement update equations as presented in Fig. 3. 
The time updated equations are responsible for projecting forward the current state and error covariance estimates to obtain a priori estimates for the next time step. The measurement updated equations are responsible for the feedback which incorporating a new measurement into the a priori estimates to obtain an improved a posterior estimate (Vu et al., 1999; 2001; Warland and Arne, 2001; Chen et al., 2012). The update time is actually the prediction stage of the DKF while the measurements update represented the correction stage. Indeed, the final estimation algorithm resembles that of a predictor-corrector algorithm for solving numerical problems (Bishop and Welch, 2001).

\subsection{Extended Kalman Filter (EKF)}

The EKF able to linearize the estimation around the current estimate using the partial derivatives of the procedure and measurement functions to compute estimates even in the face of non-linear relationship. As with the basis $\mathrm{DKF}$, the measurement updated equations correct the state and covariance estimates with the measurement, $\mathrm{Z}_{\mathrm{k}}$. An important feature of the EKF is that the Jacobian, $\mathrm{H}$ in the equation for the Kalman gain, $\mathrm{K}_{\mathrm{k}}$ serves to correct and propagate only the relevant component of the measurement information as provided in Fig. 4 (Bishop and Welch, 2001).

\subsection{Unscented Kalman Filter (UKF)}

The UKF is founded on the intuition that it is easier to approximate a probability distribution that it is to approximate an arbitrary non-linear function or transformation. The complete process diagram is provided in Fig. 5. The sigma points are chosen so that their mean and covariance to be exactly $\mathrm{x}_{\mathrm{k}-1}{ }^{\mathrm{a}}$ and $\mathrm{P}_{\mathrm{k}-1}$.

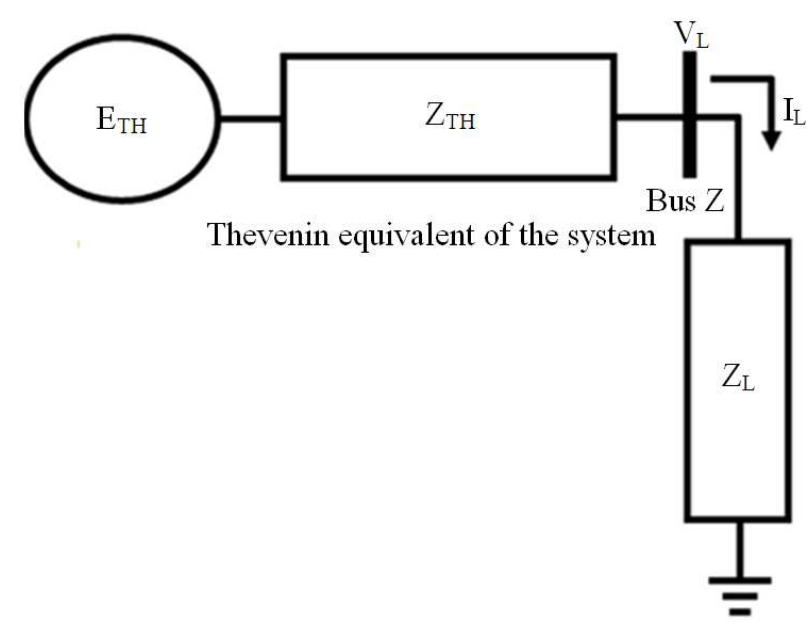

Fig. 2. Thevenin equivalent network
Each sigma point is then propagated through the nonlinearity yielding in the end a cloud of transformation points. The new estimated mean and covariance are then computed based on their statistics. This process is called unscented transformation. The unscented transformation is a method for calculating the statistics of a random variable which undergoes a non-linear transformation.

\section{Time Update:}

1. Project the state ahead.

$$
\hat{x}_{k}{ }^{-}=A \hat{x}_{k-1}{ }^{-}+B u_{k-1}
$$

2. Project the error covariance ahead.

$$
P_{k}{ }^{-}=A P_{k-1} A^{T}+Q
$$

\section{Measurement Update:}

1. Compute the Kalman gain.

$$
K_{k}=P_{k}{ }^{-} H^{T}\left(H P_{k}{ }^{-} H^{T}+R\right)^{-1}
$$

2. Update estimate with measurement, $Z_{k}$.

$$
\hat{x}_{k}=\hat{x}_{k}{ }^{-}+K_{k}\left(z_{k}-H \hat{x}_{k}{ }^{-}\right)
$$

3. Update the error covariance.

$$
P_{k}=\left(I-K_{k} H\right) P_{k}^{-}
$$

Fig. 3. A complete operation of the DKF for the Thevenin equivalent estimation process

\section{Time Update:}

1. Project the state ahead.

$$
\hat{x}_{k}^{-}=f\left(\hat{x}_{k-1}, u_{k-1}, 0\right)
$$

2. Project the error covariance ahead.

$$
P_{k}{ }^{-}=A P_{k-1} A_{k}{ }^{T}+W_{k} Q_{k-1} W_{k}{ }^{T}
$$

\section{Measurement Update:}

1. Compute the Kalman gain.

$$
\begin{gathered}
K_{k}=P_{k}{ }^{-} H_{k}{ }^{T}\left(H P_{k}{ }^{-} H_{k}{ }^{T}+V_{k} R_{k} V_{k}{ }^{T}\right)^{-1} \\
\text { 2. Update estimate with measurement, } z_{k} . \\
\hat{x}_{k}=\hat{x}_{k}{ }^{-}+K_{k}\left(z_{k}-h\left(\hat{x}_{k}{ }^{-}, 0\right)\right) \\
\text { 3. Update the error covariance. } \\
P_{k}=\left(I-K_{k} H\right) P_{k}{ }^{-}
\end{gathered}
$$

Fig. 4. A complete operation of the EKF for the Thevenin equivalent estimation process 
Initialization of $\hat{x}_{0}$ and $P_{0}$ then calculate sigma points:

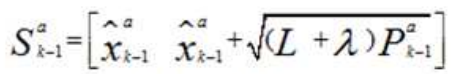

$$
\begin{aligned}
& \text { Time update: } \\
& S_{k=-1}^{x}=F\left[S_{k-1}^{x} S_{k-1}^{v}\right] \\
& \hat{x}_{k}=\sum_{i=0}^{2 L} W_{i}^{(m)} S_{i: \mathrm{k}_{-1}-1}^{x} \\
& P_{k}^{-}=\sum_{i=0}^{2 L} W_{i}^{(c)}\left[S_{i, k \mid k-1}^{x}-\hat{x}_{k}^{-}\right]\left[S_{i, k \mid k-1}^{x}-\hat{x}_{k}^{-}\right]^{T} \\
& z_{\text {k }-1}=H\left[S_{k k-1}^{x} S_{k-1}^{n}\right] \\
& \hat{z}_{k}=\sum_{i=0}^{2 L} W_{i}^{(m)} z_{i, k j-1}
\end{aligned}
$$

Measurement Update:

$$
\begin{gathered}
P_{z_{k} z_{k}}=\sum_{i=0}^{2 L} W_{i}^{(c)}\left[z_{i, k \mid k-1}-\hat{z}_{k}^{-}\right]\left[z_{i, k \mid k-1}-\hat{z}_{k}^{-}\right]^{T} \\
P_{x_{k} z_{k}}=\sum_{i=0}^{2 L} W_{i}^{(c)}\left[S_{i, k \mid k-1}-\hat{x}_{k}^{-}\right]\left[z_{i, k \mid k-1}-\hat{z}_{k}^{-}\right]^{T} \\
K_{k}=P_{x_{k} z_{k}} P_{z_{k} z_{k}}^{-1} \\
\hat{x}_{k}=\hat{x}_{k}^{-}+K_{k}\left(z_{k}-\hat{z}_{k}^{-}\right) \\
P_{k}=P_{k}^{-}-K_{k} P_{z_{k} z_{k}} K_{k}^{T}
\end{gathered}
$$

Fig. 5. A complete operation of the UKF for the Thevenin equivalent estimation process

\subsection{Voltage Stability Monitoring Indicator}

By incorporating the estimated Thevenin impedance value from different Kalman filter implementations, the minimum magnitude of the load impedance in a stable system can be determined. Hence, the monitoring index can be defined in Equation 3 to invigorate the condition of the current power system:

Index impedance $=\frac{\mid \mathrm{Z} \text { app }|-| \mathrm{Z} \text { th } \mid}{\mid \mathrm{Z} \text { th } \mid}$

Additional power will be spent whenever the load impedance started to sink. By this, the monitoring index also decreases indicating that the system is an edge near to the nose point of voltage collapse. The index definitely becomes zero when the system is operating from the nose point. The main perception is to ensure the index always stays positive and suitable early prevention should be implemented when the index is close to zero.

\section{RESULTS AND DISCUSSION}

For more detailed analysis, the application is tested with simulation data from Real-Time Digital Simulator (RTDS). This is to ensure that voltage instability would occur in the specified load area and in the meantime to estimate the performance of RTVSM application when the system is exposed voltage instability phenomena.

\subsection{Employment of TNB $132 \mathrm{kV}$ Network (2 Bus and 10 Bus Systems)}

The TNB $132 \mathrm{kV}, 2$-bus and 10-bus systems are employed to examine the ability of the three different Kalman filter implementations in order to test the strength of the voltage stability margin. The $132 \mathrm{kV}$ network, 2-bus and 10-bus systems of the research area, where the incoming $275 \mathrm{kV}$ supply is taken from Bus 1 and stepped down to $132 \mathrm{kV}$ by two units of 180 MVA transformers, which connected to Bus 1 and Bus 2. All buses in the system are considered as the load bus. Load bus can be defined as transmission line feeding a certain load. PMUs are installed to monitor voltage and current phasors from Bus 1 up to Bus 10 .

The PMUs data provided for 2-bus system takes in a total number of 32973 data points while for 10-bus system has a total number of 2000 data points. Besides, both systems also consist a same step size of $0.02 \mathrm{sec}$ which means that the PMUs able to provide 50 samples per second. The TNB $132 \mathrm{kV}$ load area under research for 2-bus and 10-bus systems are provided in Fig. 6.

\subsection{Event A: Results for 2 Bus System}

In relation to the given data, the set of data in 2 bus system shows an abnormal phenomenon at time 634.1 seconds; thus, a prediction that the degree of voltage collapse potentially happens at time $634.1 \mathrm{sec}$. The results of the three methods which are DKF, EKF and UKF are shown in Fig. 7-9 respectively.

\subsection{Event B: Results for 10 Bus System}

In the meanwhile for the 10 bus system, the foretelling of the degree of voltage collapse is at time $0.16 \mathrm{sec}$. The results of the three methods of DKF, EKF and UKF are shown in Fig. 10-12 respectively.

\subsection{Results for the Implemented Voltage Stability Monitoring Index}

The indices of the early warning for point of voltage collapse are calculated based on the estimated Thevenin impedance by using different Kalman filtering implementations is shown in Table $\mathbf{1}$. 


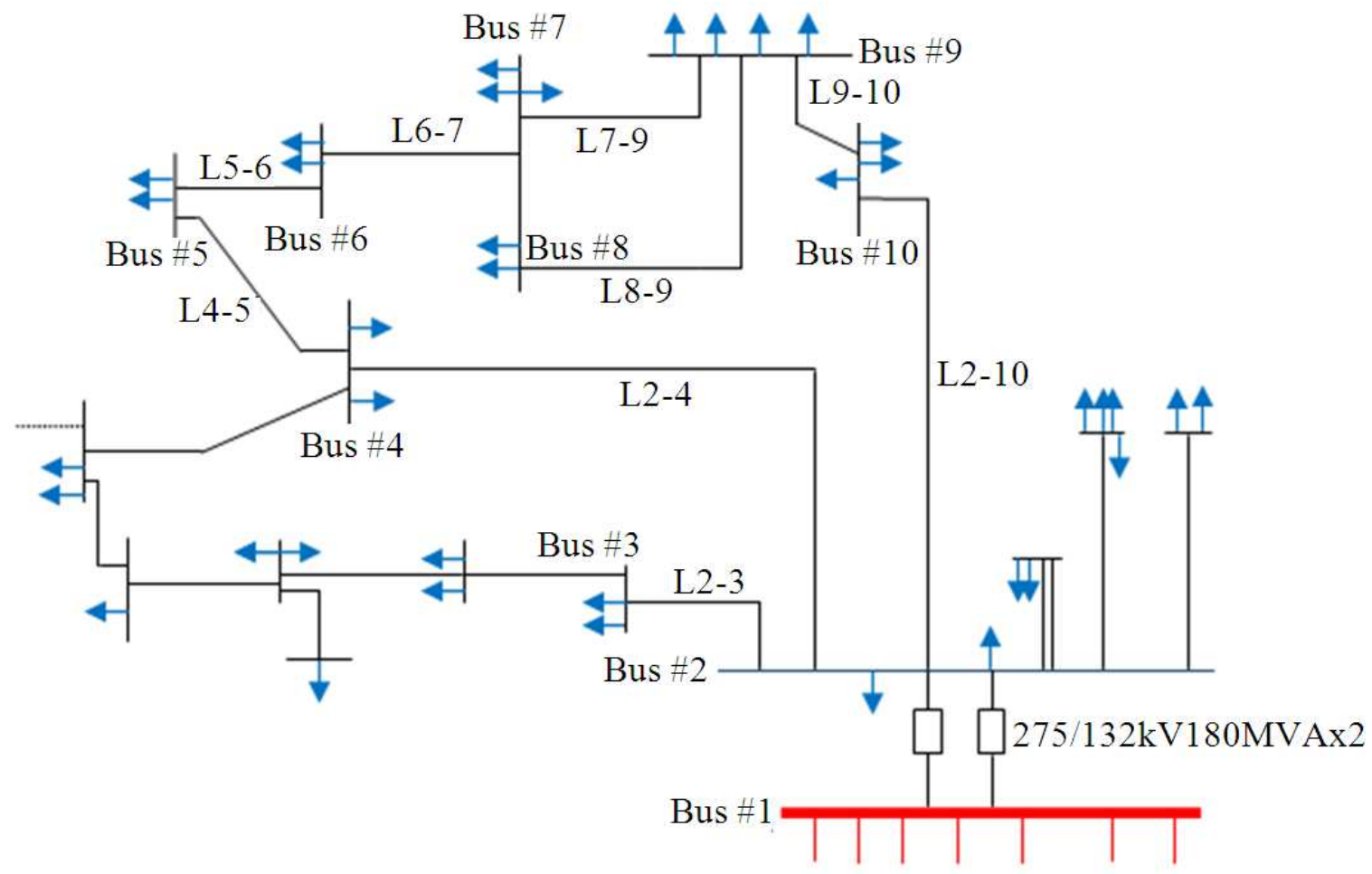

Fig. 6. Detail of TNB $132 \mathrm{kV}$ load area under investigation

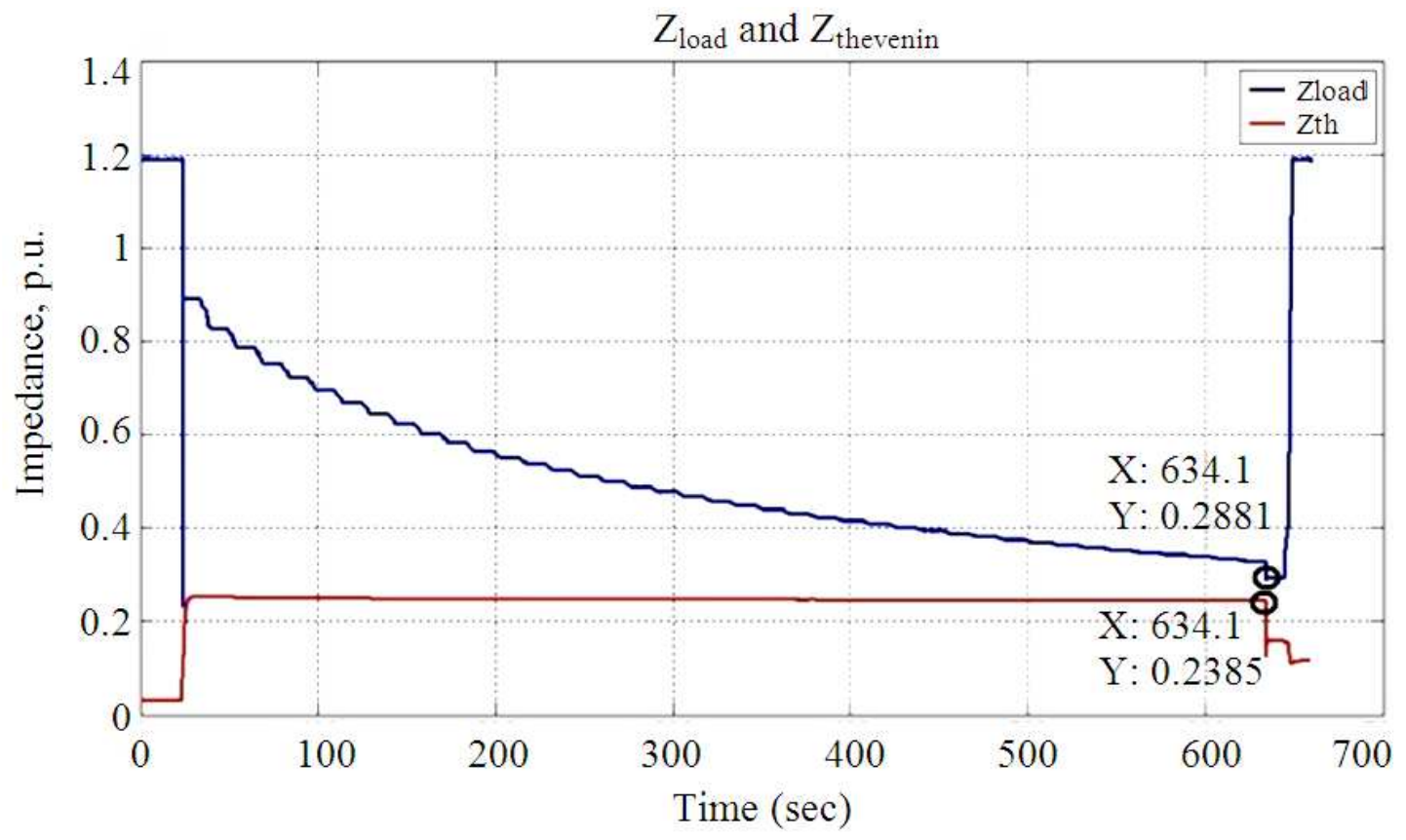

Fig. 7. Graph of load impedance with Thevenin impedance by DKF approach 


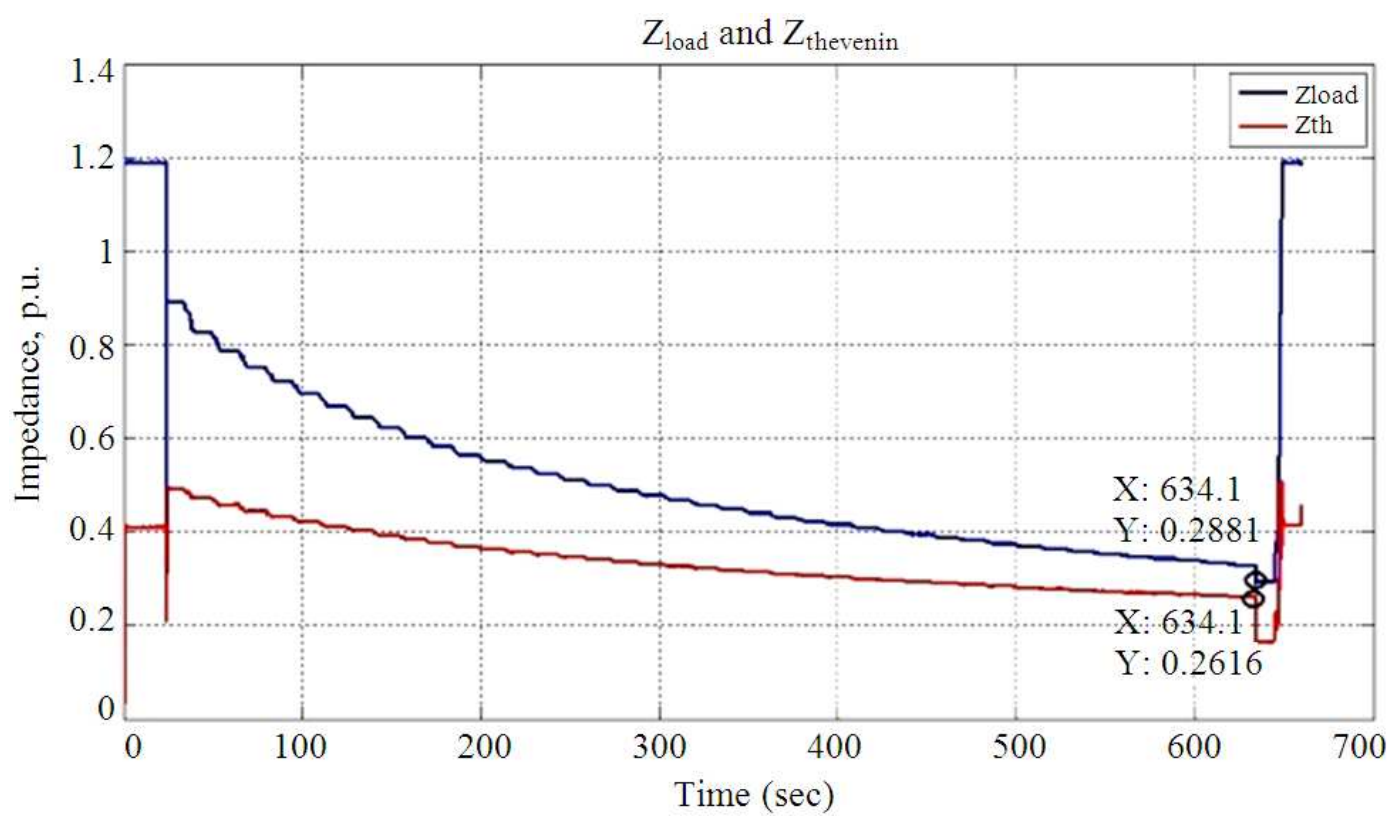

Fig. 8. Graph of load impedance with Thevenin impedance by EKF approach

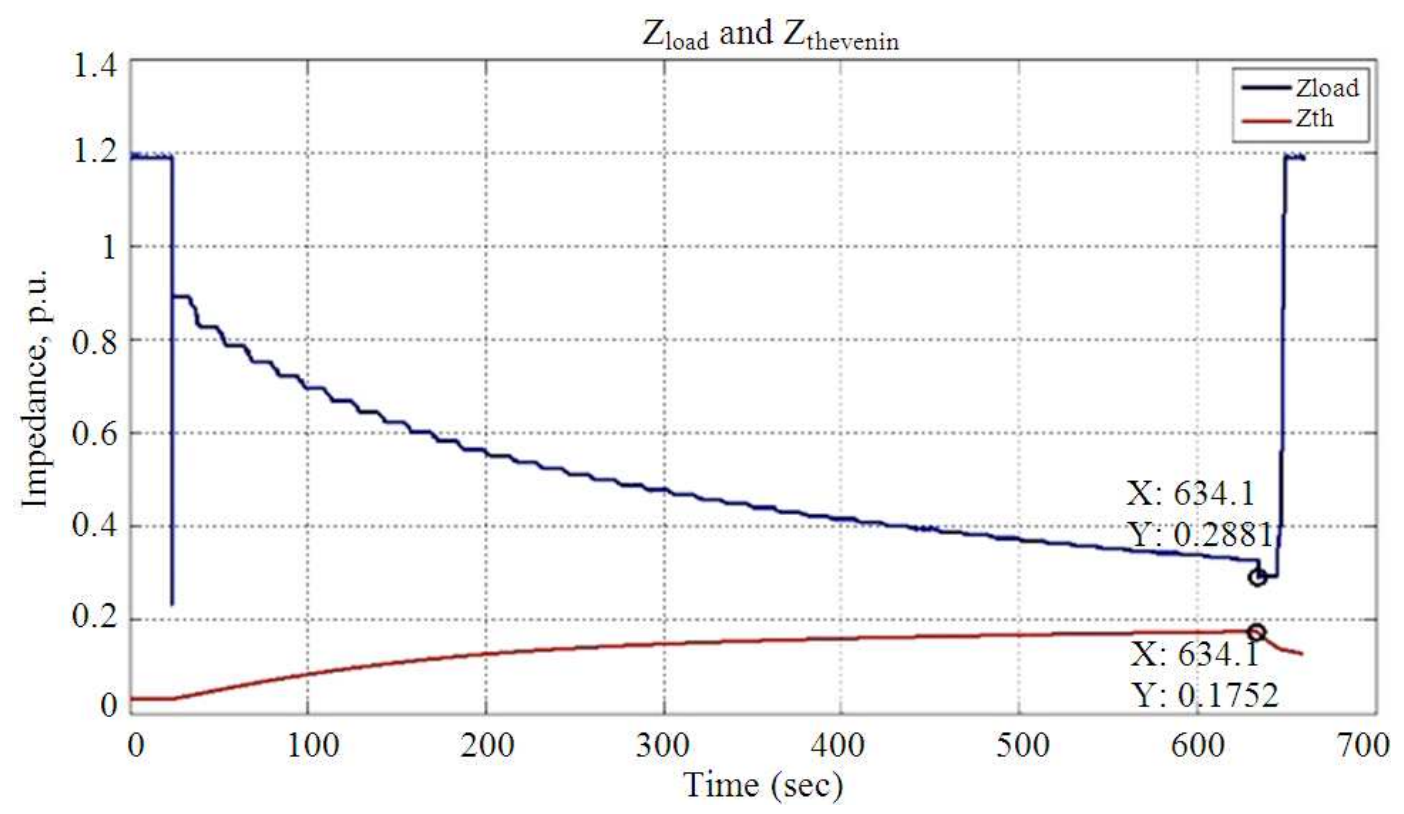

Fig. 9. Graph of load impe dance with Thevenin impedance by UKF approach

\section{DISCUSSION}

\subsection{Event A: Discussion for 2 Bus System}

The magnitude of load impedance, $\mathrm{Z}_{\text {load }}$ which is 0.2881 p.u. remained constant for the graphs. In the meanwhile, the Thevenin impedances, $Z_{\text {th }}$ are varied. The magnitude for Thevenin impedance in Fig. 7 is 0.2385 p.u. and the magnitude in Fig. 8 is shown as 0.2616 p.u. Besides, the magnitude in Fig. 9 is 0.1752 p.u. The results have also proven that the point of voltage collapse of the 2 bus system is occurring at a time of 634.1 
seconds. As stated by the theory, the point of collapse occurs whenever the Thevenin impedance is equal to the load impedance for the power system network ( $\mathrm{Vu}$ et al., 2001; Warland and Holen, 2001).

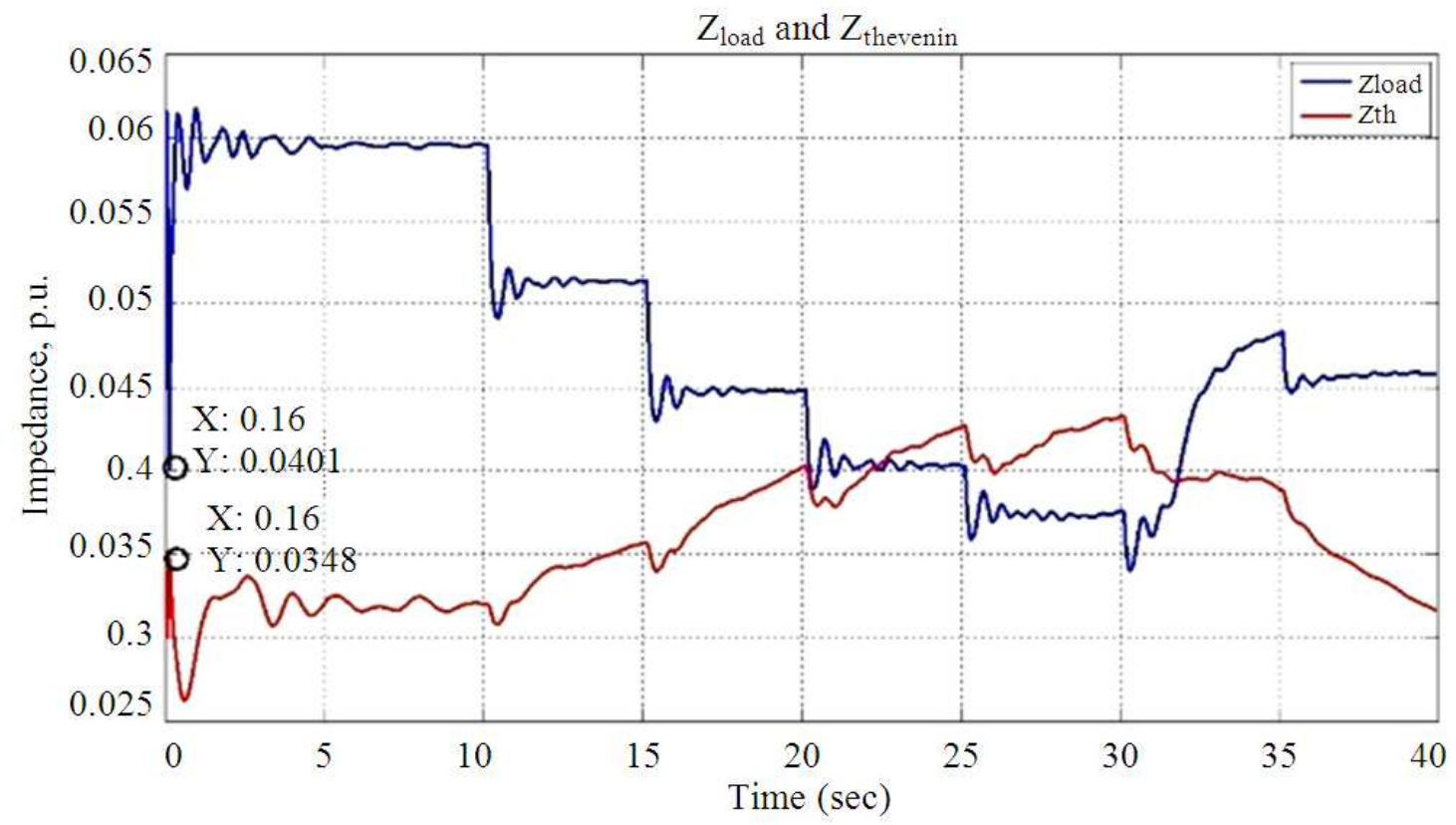

Fig. 10. Graph of load impedance with Thevenin impedance by DKF approach

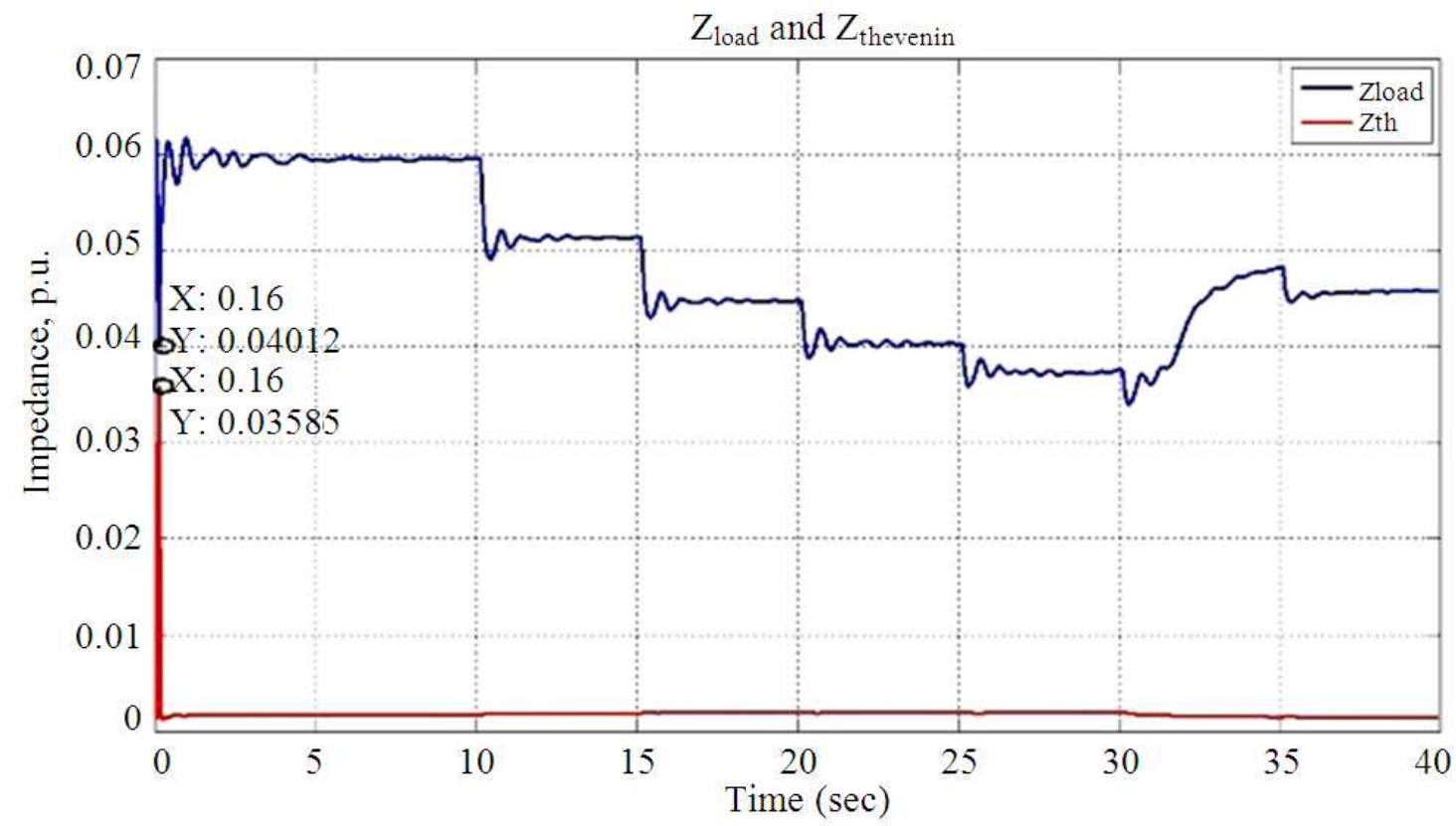

Fig. 11. Graph of load impedance with Thevenin impedance by EKF approach 


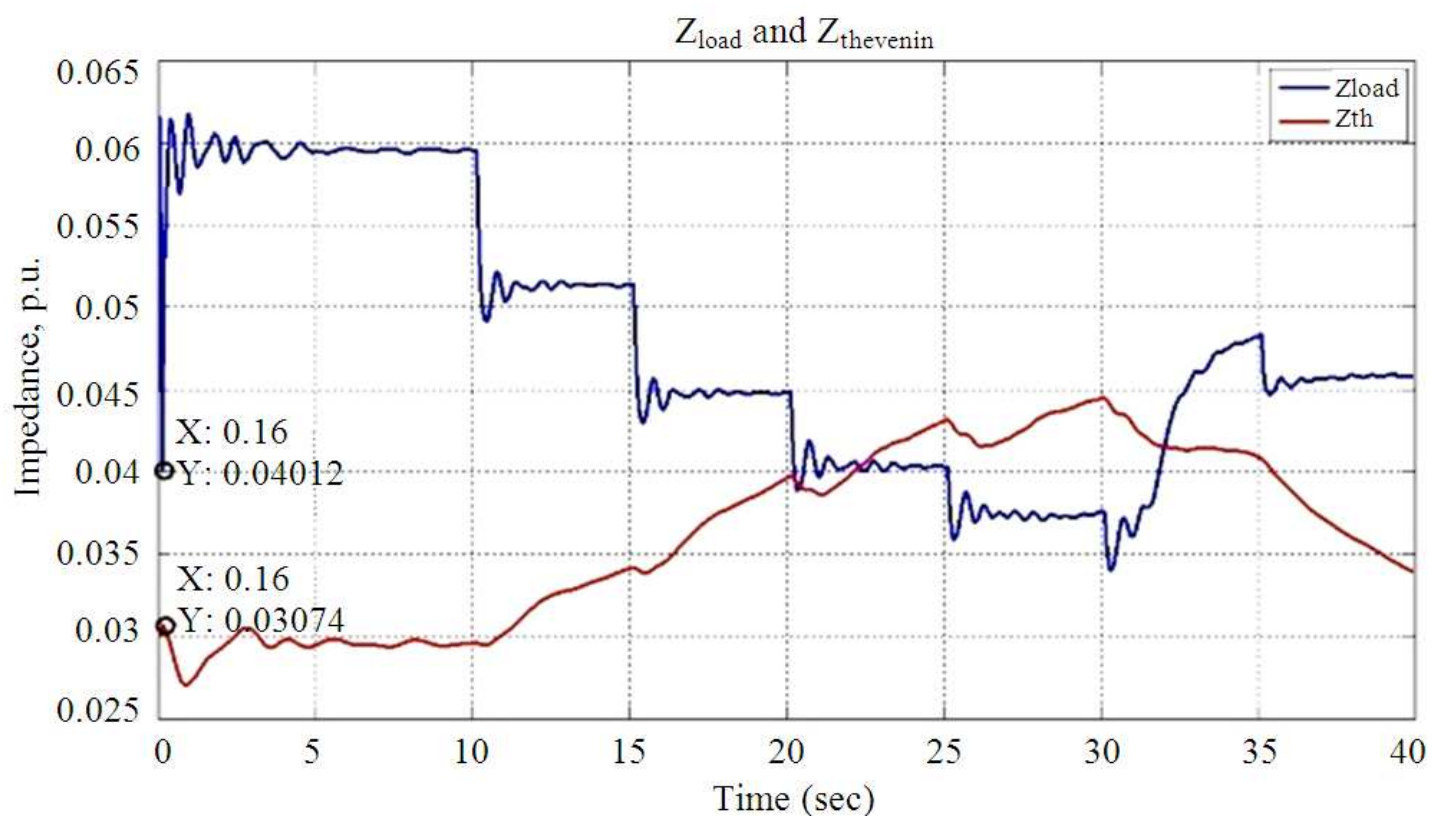

Fig. 12. Graph of load impedance with Thevenin impedance by UKF approach

Table 1. Monitoring index for voltage collapse

\begin{tabular}{llr}
\hline & Index for early warning & \\
& - & \\
Types of method & 2-bus system & 10-bus system \\
\hline $\begin{array}{l}\text { Discrete Kalman } \\
\text { Filter algorithm (DKF) }\end{array}$ & 0.2182 & 0.1529 \\
$\begin{array}{l}\text { Extended Kalman } \\
\text { Filter algorithm (EKF) }\end{array}$ & 0.1013 & 0.1191 \\
$\begin{array}{l}\text { Unscented Kalman } \\
\text { Filter algorithm (UKF) }\end{array}$ & 0.6444 & 0.3051 \\
\hline
\end{tabular}

\subsection{Event B: Discussion for 10 Bus System}

The magnitude of load impedance, Zload which is 0.0401 p.u. remained constant for the graphs. In the meanwhile, the Thevenin impedances, Zth are varied. The magnitude for Thevenin impedance in Fig. 10 is 0.0348 p.u. and the magnitude in Fig. 11 is shown as 0.0358 p.u. Likewise, the magnitude in Fig. 12 is 0.0307 p.u. The results also have also proven that the point of voltage collapse of the 10-bus system is happening at a time of 0.16 seconds. According to the theory, the point of collapse occurs whenever the Thevenin impedance is equal to the load impedance for the power system network. The system will be a normal state if the Thevenin impedances are smaller (or very much smaller) than the load impedance (Vu et al., 1999).

\subsection{Discussion for the Implemented Voltage Stability Monitoring Index}

By comparing the values of the index, it is very obvious that the result for the EKF is much more accurate because of its value for the index is closer to zero. Thus, we concluded that the EKF algorithm performs better in tracking the points of collapse in the 2 bus and 10 bus systems and then follows by the KF algorithm and lastly, the UKF algorithm.

The overall discussion is based on the performance of three different types of Kalman filter methods for tracking the Thevenin parameters. In the way of only looking at its performance to trace the points of voltage collapse, which basically only refer to its Thevenin impedance and load impedance, the EKF performs the best among them. However, the results of estimating the Thevenin voltage from EKF are divaricated. This phenomenon may due to the large error in the true posterior mean and covariance. As the EKF is suitable for non-linear system and we presented the Thevenin parameters in its rectangular frame in order to take on the non-linear requirement.

Therefore, both KF and EKF can also be used to track the Thevenin parameters (Vu et al., 1999; 2001; Warland and Holen, 2001; He et al., 2011; Chen et al., 2012). If comparing the results of the KF with the $\mathrm{EKF}$, the overall performance is better in the EKF 
algorithm. The performance of EKF for its Thevenin voltage can actually be addressed by using the UKF. The overall performance of the UKF in this research can be considered as moderate and it does perform better than the EKF in the Thevenin voltage prediction. However, the UKF is slightly more complex in its level of complexity. It is more suitable for the highly non-linear system, which involves a higher level of order in the state vector.

The results are compared based on the early-warning index of voltage collapse. The results of DKF method are set as the reference for comparison purpose between EKF method and UKF method. The test results show that EKF method provided better results by decreasing of 0.1169 p.u. for 2 bus system and 0.0338 p.u. for 10 bus system. In the mean while, UKF method provided increasing values of 0.4262 p.u. for 2 bus system and 0.1522 p.u. for 10 bus system. The overall aim of this innovation is to produce methods that offer early warning of an emerging stability problem. In order to achieve the invention's objectives, derivation of the index for early warning of the point of collapse is completed. The performance of each method used throughout this invention is based on the analysed results for the points of voltage collapse.

\section{CONCLUSION}

The overall aim of this research was to develop methods that in real-time provide an early warning of an emerging stability problem. It was interesting to develop on methods of tracking the points of voltage collapse of the electric power system and then give an early warning for the emerging blackout or voltage collapse. In parliamentary law to achieve the research's objectives, derivation of the index for early warning of the point of collapse is done. There are three methods, which have been employed for this online estimation of the model parameters which are the KF, EKF and UKF. Comparisons of the results and performance of all of these methods have also been made in section 4. As a conclusion, the presented methods fulfill the requirements. This means that a development of the high accuracy for PMUs measurements could be achieved. The results make the presented assessment method an attractive instrument for real-time stability assessment in future prevention of voltage collapse.

The proposed methods are quite promising for real-world application. Further studies can be focused on a larger system such as the IEEE 14-bus and 118- bus in order to support the performance of the Kalman filters in predicting the Thevenin equivalent.

\section{ACKNOWLEDGEMENT}

The researchers gratefully acknowledge the contributions of TNB Research and TNB Transmission members for their valuable comments, support and technical cooperation during the process of collecting the PMUs data and also the development works. The authors would like to thank the Ministry of Energy, Green Technology and Water Malaysia (KeTTHA), Ministry of Higher Education, Malaysia (MOHE) and The Office for Research, Innovation, Commercialization, Consultancy Management (ORICC), UTHM for financially supporting this research under the Fundamental Research Grant Scheme (FRGS-KeTTHA) grant No.0905 and MTUN-COE Vot.No.C025.

\section{REFERENCES}

Ajjarapu, V. and A.P.S. Meliopoulos, 2008. Preventing voltage collapse with protection systems that incorporate optimal reactive power control. Power Syst. Eng. Res. Center.

Al-Ammari, R.A., 2002. Voltage stability margin identification using local measurements and linear kalman filter. Eng. J. Univ. Qatar, 15: 153-164.

Balasubramanian, R. and R. Singh, 2011. Power system voltage stability analysis using ANN and continuation power flow methods. Proceedings of the 16th International Conference on Intelligent System Application to Power Systems, (SAP' 11), IEEE Xplore Press, pp: 1-7. DOI: 10.1109/ISAP.2011.6082192

Bishop, G. and G. Welch, 2001. An introduction to the kalman filter. Proc. SIGGRAPH, Course, 8: 2759-3175.

Chakrabarti, A. and S. Halder, 2006. Power System Analysis: Operation And Control. 1st Edn., PHI Learning Pvt. Ltd., ISBN-10: 9788120327771, pp: 660.

Chen, J., L. Min and P. Zhang, 2012. Measurement based voltage stability monitoring and control. U.S. Patent 8126667, Washington, DC: U.S. Patent and Trademark Office.

Ghahremani, E. and I. Kamwa, 2011. Online state estimation of a synchronous generator using unscented Kalman filter from phasor measurements units. IEEE Trans. Energy Convers., 26: 1099-1108. DOI: $10.1109 /$ TEC.2011.2168225 
He, H., R. Xiong, X. Zhang, F. Sun and J. Fan et al., 2011. State-of-charge estimation of the lithium-ion battery using an adaptive extended Kalman filter based on an improved Thevenin model. IEEE Trans. Vehicular Technol., 60: 1461-1469. DOI: 10.1109/TVT.2011.2132812

Kundur, P., 1994. Power System Stability and Control. 3rd Edn., CRC Press, ISBN-10: 1439883203, pp: 450.

Kundur, P., J. Paserba, V. Ajjarapu, G. Andersson and A. Bose et al., 2004. Definition and classification of power system stability IEEE/CIGRE joint task force on stability terms and definitions, power systems. IEEE Trans., 19: 1387-1401. DOI: 10.1109/TPWRS.2004.825981

Lee, B.H. and K.Y. Lee, 1991. A study on voltage collapse mechanism in electric power systems. Trans. Power Syst., 6: 966-974. DOI: $10.1109 / 59.119236$

Min, L., N. Zhang and P. Zhang, 2009. Method for voltage instability load shedding using local measurements. U.S. Patent No. 7603203. Washington, DC: U.S. Patent and Trademark Office.

TNB, 2011. Announcement on the Increase in Electricity Tariff in Peninsular Malaysia Due to the Revision of Nature Gas Price. Revision of Base Tariff and Implementation of Feed-In-Tariff (FiT) for Renewable Energy (RE).

Vaiman, M., K. Bell, Y. Chen, B. Chowdhury and I. Dobson et al., 2012. Risk assessment of cascading outages: Methodologies and challenges. IEEE Trans. Power Syst., 27: 631-641. DOI: 10.1109/TPWRS.2011.2177868
Vu, K., M.M. Begovic, D. Novosel and M.M. Saha, 1999. Use of local measurements to estimate voltage-stability margin. IEEE Trans. Power Syst., 14: 1029-1035. DOI: 10.1109/59.780916

Vu, K.T., D.E. Julian, J.O. Gjerde and M.M. Saha, 2001. Applications and methods for Voltage Instability Predictor (VIP). U.S. Patent No. 6249719, Washington, DC: U.S. Patent and Trademark Office.

Warland, L. and H.T. Arne, 2001. A voltage instability predictor using local area measurements (VIP++). Proceedings of the IEEE Porto Power Tech Proceedings, Sept. 10-13, IEEE Xplore Press, Porto. DOI: 10.1109/PTC.2001.964729

$\mathrm{Wu}, \mathrm{H}$. and J. Giri, 2006. PMU impact on state estimation reliability for improved grid security. Proceedings of the Transmission and Distribution Conference and Exhibition, May, 21-24, IEEE Xplore Press, Dallas, TX, pp: 1349-1351. DOI: 10.1109/TDC.2006.1668709

Zhu, K., L. Nordstrom and L. Ekstam, 2009. Application and analysis of optimum PMU placement methods with application to state estimation accuracy. Proceedings of the Power and Energy Society General Meeting, Jul. 26-30, IEEE Xplore Press, Calgary, AB, pp: 1-7. DOI: 10.1109/PES.2009.5275241 